\title{
did $\mathfrak{n} \mathfrak{i} \mathfrak{e} \mathfrak{d}$
}

Das Sehrbu币, meldeses hiermit in einer bođftändig neuen Bearbeitung erfheint, ging uriprïnglid aus ber boppelten 2 bjifdt herbor, einerjeits ben praftifden $\mathfrak{J} u=$

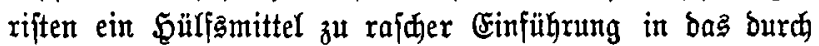

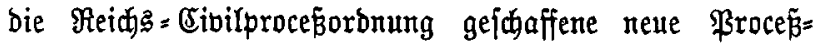

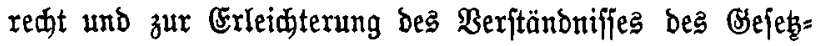
buthes zu bieten, andererfeits aber burd gemeinfap̉lide

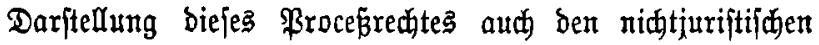
Freifen bes beutiden $\mathfrak{B o l f e s}$ eine nimb gantz oberflädhlidue

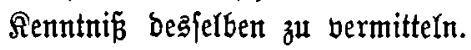

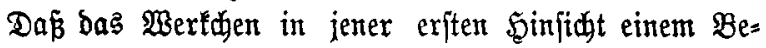
Dürfniffe entiprad, Gat fein Erfolg fattfam berwiejen. IUuc Den zmeiten Theil feiner Beftimmung hat es burdians nidt

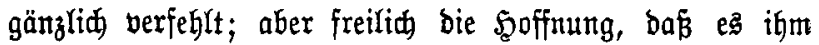

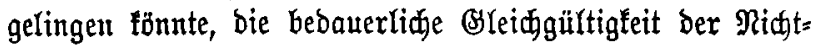
jurijten Deutfaldands für redtlide Dinge in nennenswerthem Maß̃e zu überwinden und bem neuen Civilproceffe zu einer getwiffen Bolfzthümlidjeit zu verhelfen, hat fich nidt cinmal annährento erfülat. Dafür hat es als hoch exfren= lidjen Erjak eine ftets wadjjente Berbreitung bei ben Stubirenden gefunden. 


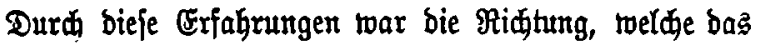
But bei einer Neubearbeitung einzujolagen habe, in ganz bejtimmter Beije vorgezeidnet. $\mathfrak{A n}$ ber bewährten Srrund: anlage jelbjt burfte niat gerüttelt, und namentlidg mub̈ten bem Merfe bie Cigeniळaften, denen ez jeine Erfolge

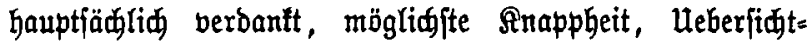
lidłfeit uno Qeidtfap̧lidfeit ber Darjtellung, forglam ge= wahrt twerber. Dagegen war Die Rüdfidht auf bie Riat= juriften bölig preiżgegeben. $\mathfrak{A}$ bie Stelle einer nađ̆ Der wiffenjळaftliaen Seite anjprumlojen arbeit mupte eine ftreng wiffenjafaftlixe Behanblung treten, ein ftetes Burüafgehen auf bie tieferen, leitenden Srundjäşe und eine fortlaufende Darlegung bes zroijhen ben einzelıen gejes. lidjen Boriduriften bejtebenden inneren $8 \mathfrak{u}$ jammenhanges.

Dak bas \&ehrbum einer Umarbeitung in biejem Simme be=

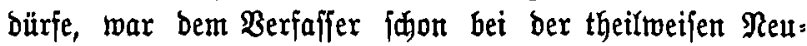
benrbeitung flar getworben, welde im Şerbjt 1879 als vierte

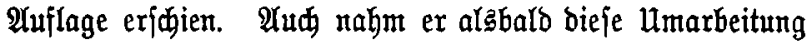
in Angriff. Sie ertwies fich aber bei ber 2 uafiithrung als

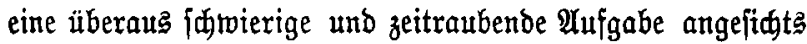
ber hödjt umfangreiden Qitteratur, welde rajd auf (Jrund ber Civilproceporbnung ermuds, und ber zahllojen Streit= fragen, welde fid nidt nur über ben Sinn ber einzelnen gefeblichen Borjdriften, fonbern jogar über die tiefiten

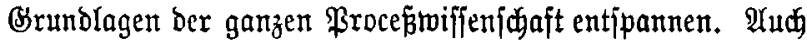

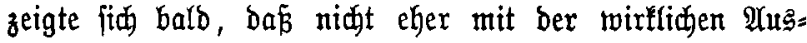
arbeitung bes Buhes in ber beabfintigten neuten Beftalt begonnen werben fönne, als bis burd jorgfärtigftes Stu= bium jener Sitteratur, namentidi aber burm genauejte

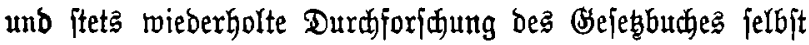
für bie riftige Bebandlung im Sanzen wie in allent 


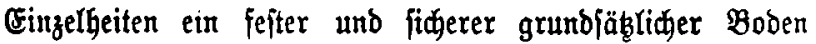
gewonten jei.

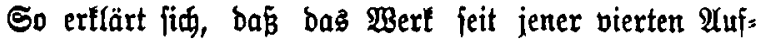
lage unb länger als zeghn ̧ăahre völlig unverändert blieb, um bafür jeķt in bejto gründridferer Umgejtaltung an bie

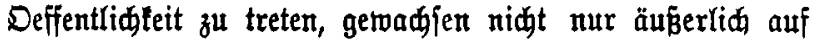
Das Doppelte bes bişherigen Umfanges, fondern ungleid mehr nod innerlid an wifjenjdaftlider Bertiefung, Bodl=

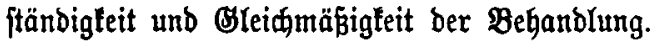

Während bie biăherigen $\mathfrak{A}$ uflagen, wie bas untmittelbar

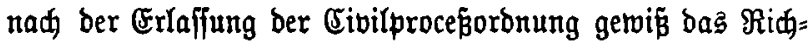
tige war, fi் vorzugâmeife an bic Begrünoung und an bie Urbeiten joldyer Männer anjdlofjen, melde bei ber $\mathfrak{A} \mathfrak{b}$,

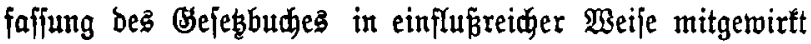
hatten, hat bie gegenmärtige Neubearbeitung eine vößig freie und jelbftänoige Stellung genommen, - eine Stellung, welde Gie und ba, namentlid im Şinblid auf bie abrweidjende

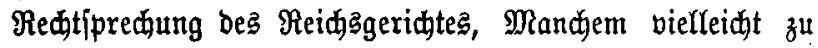
frei erideinen mag. Indejifen, wer bie genannte Recht= iprecdung näher verforgt hat, ber meif́, wie wenig hier überall now bon irgend einer befejtigten Pracis die Rede jein fanm. Und ez eridjeint baher midjt allein als ein gutes

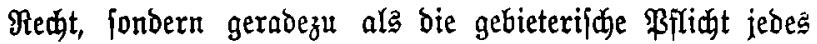

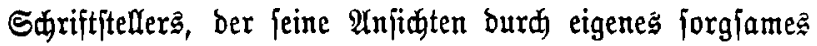
Stubium gewonnen hat, fith aud nadi biejer Seite bie volle Selbjtändigkeit zu rahren. Für bas vorliegende Werf fonnte fie jifon barum gar nidft aufgegeben werben, weil jonjt zugleid) auf jede (Einheitfliduleit Der Darịtellung hätte verzidytet werben mínjen.

Banz bejondere Sorgfalt ift bei der neuen Bearbeitung dent Spradgebraude des Gejeę̧udues gemiomet morocn, 
Borrebe.

ba feine Reuntniß unb genaute Peahtung bie erfte unb notgwenbigfte Borbebingung für bas riøtige Berftänoni ber gefestiden Boridriften if. Nidt nut bebient fing bas Rebrbud felbft iberall biefes Spradgebraumes, fonbern bie gejegfífen Sumftaubbrüde finb aud entroeber ba, too fie zum eriten ERal auftreten, ober ba, wo igre Bebeutung erläutert toirb, burd Infübrungsizeiden lenntlid gemadt und fo

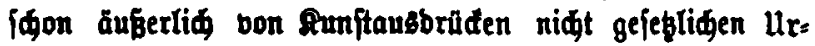
iprumges unterjøieben. In gleidger Eseife ift aud fonit mitunter auf ben $\$$ ortlaut be Sejeges bingetwielen.

\$egen ber naben Bejiebung biejes Legrbuđeş zu bem

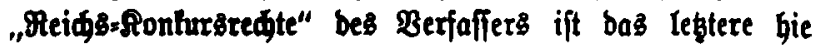
unb ba angeführt. Im Uebrigen ift von Berweijungen auf bie Ritteratur, wie fie in ber bierten Pluflage in einem ge= wiffen, allerbings pparjamen, Maß́be auftreten, roiebenum, wie in ben brei erften Euflagen, ganz abgejeben. Denn eine blope Nennung bon Bertretern übereinjtimmenber ober

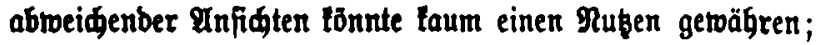
eine fortlaufenbe Eubzeinanberję̧ung aber unit ber bunten Prannigfaltigleit ber in ber Sitteratur aufgetaumten Mei= nungen unb ihren Srūnben bätte ungemein viel Faum er: forbert unb bie Beruşung bes Segrbudes als folden

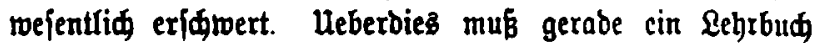
allentbalben burd fi் felbjt verftändlid fein. Das viro

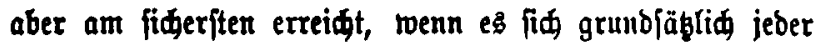
Sintoeifung auf anbere Śfriften, fogar auf bie eigenen feines Berfalier entbält.

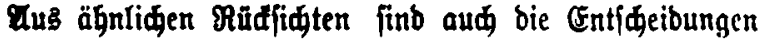

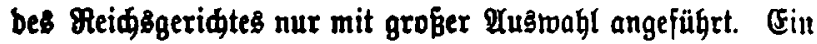

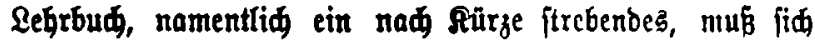
überbaupt notgroendig befकränten und Iemjenigen, bcr fid 
nod weiter unterriøten will, bie Benukung anberer Sulfs: mittel aberlafien. Cerabe fir bie Belegnung ifber ben Stanb ber Ritteratur unb ber Fedtipredung feglt es ja aber an SulfsmitteIn nidht. Seber bee größeren Commen: tare gibt bariber eine genügenbe Gushunft, unb als bes jonbers voufanbig gerabe blerin tann berjenige bon $\mathrm{St} \mathrm{r}$ u $\mathrm{d}$ mann unb Rod (jebt 5. Fuff. 1887) becborgeboben werben.

Der Berfaffer trug fí eine Beit lang mit bem \$lan, neben ber Reubearbeitung bes \&ebrbuhe nod ein größeres

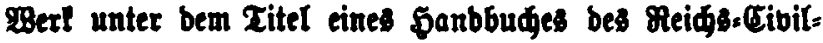
proceffes zu 伍reiben. Darauf bat er jest nad reiffider Erroägung bergidtet. Seine Brunbanfidten finb in ber neuen Bearbeitung bes \&egrbuges genugfam bargetegt. Gollte fí igm bas Beburfnif eingegenberer Crōterung einzelner \&ebren ober Fragen ergeben, fo tann es leidt burd monographifळe Beganblung in einer wiffenidaftidien Beifjorift befriebigt werben.

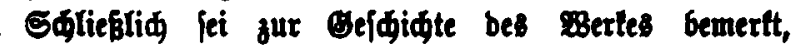
baß bie exfte (giemlid farte) Y्रuflage zu Xnfang Dttober 1878 , bie zweite wenige 280 ben ipäter, ebenfalls nod im Jabre 1878 ausgegeben murbe. Im frrüjagr 1879 folgte bie britte, in Serbft biefes Эahres bie bierte, neu bes arbeitete. Die fünfte unb fechfte (beibe unveränberte $\mathbb{Q b}=$ briide ber bierten) ex[đienen in ben Jagren 1880 unb 1884.

Galle in expril 1890. 